\title{
The scientific problem of plastic pollution: an ocean of reports with little standardization of procedures.
}

\author{
El problema científico de la contaminación plástica: un océano de informes con poca estan- \\ darización de procedimientos.
}

Bionatura guest editors: Alonzo Alfaro-Núñez $z^{1-2}$ and Rafael Bermúdez $z^{3-4}$.

DOI. 10.21931/RB/2018.03.03.1

A $s$ any other new area of research during its genesis, the study of plastic pollution in the oceans currently lacks an international consensus on procedures to ensure standardization of results. When a phenomenon, problem or issue firstly appears or it is discovered and recognized, there is no guideline, protocol or general approach on how to measure, quantify or evaluate it. More importantly, there is no general agreement on how to transmit this new knowledge in order to standardize results across research groups, states and regions. This is exactly the current state of knowledge generated around the subject of plastic pollution in the environment, and more specifically in the oceans.

To aggravate even more the lack of a standardized protocol for plastic pollution detection and quantification, today's social media have the "absolute power" to deliver information to the general public. As such, social networks turn news feed viral in minutes; millions of people can receive this information, which is then cognitively transformed into facts. Very few questions are raised as to whether or not this new information has actually been scientifically proved. As such, news in social media becomes more believable than science itself.

Plastic pollution is indeed a global problem, especially in the oceans as it has repeatedly been reported ${ }^{1-3}$. The real problem lies in the fact that there are so many scientific and non-scientific reports with different procedures following a myriad of protocols and methodologies, and so far no one can assertively attest as to what is accurate. All of the current reports, at the same time, claim to deliver new procedures with results that provide evidence to the dispersal of plastic particles and how they may dissolve in the oceans.



Figure 1. Plastic products are currently a threat to the marine environment, as they break up into micro and nano plastic particles into the oceans (Photo art by Allan Jeffs).
To assess plastic pollution has become a popular subject. In many ways it seems kind of obvious and simple, plastic pollution is ubiquitous ${ }^{1,3,4}$. It can be found in our parks, forests, rivers, lakes and oceans; possibly also in our food, drinking water and air; thus just go and collect your own sample and analyse it, as you deem convenient. All this makes these results impossible to compare, therefore creating a fragmented pool of knowledge.

Under the current and rather confusing context of approaches, it is important to start by defining what the problem is, and what is the main issue surrounding plastic pollution in the oceans. Next, we present and explain briefly, to our understanding, some general background of the current status of plastic pollution in the marine environment.

Plastic pollution in the oceans is directly correlated with this material being inexpensive, versatile, robust and durable, which is linked to the high amounts of plastics produced, used and easily discarded.

Our current knowledge on the effects of large plastic fragments on marine diversity is still limited. Hundreds of species of fish, seabirds, turtles, and marine mammals have been documented to become entangled or to ingest pieces of plastics like ropes, fish nets, plastic bags, etc., which may result in ulceration or starvation. ${ }^{5}$. Plastic contamination contributes to the alarming estimation that at least one-third of all marine species are now threatened with extinction ${ }^{6}$. This phenomenon receives significant media coverage and usually produces shocking images of dead charismatic animals like sea turtles, whales, birds, etc., and thus it is the issue that most people are familiar with.

Although plastic can eventually disintegrate into smaller pieces down to the molecular level, it remains intact as plastic polymers (the main blocks that form and hold the plastic structure together) and attracts persistent organic pollutants (POPs) such as dioxins and 1,1-dichloro-2,2-bis (p-chlorophen$y l)$ ethylene also known as DDE, on top of the toxic chemicals they already contain (e.g. bisphenol A (BPA) and flame retardants).

Plastic polymers are not processable in their natural form; hence they are mixed with plastic additives. As such, plastic additives comprise a large number of chemical substances such as flame-retardants, surfactants, blowing agents, and plasticizers. However, most plastic additives are often not covalently bound to the original plastic polymer matrix, therefore they can slowly diffuse out of plastics leading to wide environmental contamination ${ }^{7}$, becoming POPs. These POPs compounds are toxic, bioaccumulative and subjected to long-range transportation in the ocean. The list of POPs in the Stockholm Convention consists of 20 compounds including BPA, chlorinated pesticides, chlorinated industrial chemicals, bromina- 
ted flame-retardants (BFRs) and one fluorinated surfactant. However, there are hundreds of other POPs in production, e.g. out of the 75 commercially recognized BFRs, only 3 groups are on the list; and of the more than 115 poly- and perfluorinated surfactants found in industrial blends ${ }^{8}$, only one is on the list.

Simultaneously, POPs have been suggested as a potential cofactor for the increase of infectious diseases and the large viral diversity found in marine environments ${ }^{9}$. Thus, environmental plastic contaminants may be directly involved in the dramatic decrease of marine wildlife, and the increase of viral infectious diseases in the ocean through several plausible mechanisms including carcinogenesis and contaminant-induced immune suppression; and as cofactors for reproductive and clinical failure in marine vertebrates ${ }^{10}$. Furthermore, POPs might cause endocrine disruption and toxicity to all levels of taxonomic groups in mammals and non- mammalian marine species ${ }^{11}$.

Among the POPs, BPA is just the best-documented "bad guy" with a large record of scientific publications documenting its effects. BPA, however, may only represent a microscopic fraction of the real complex mixture image of chemicals involved. Hence, we might only have seen the tip of the iceberg; many more organic chemicals are used in our society for plastic production and many of them can be detected both in the environment, wildlife and humans.

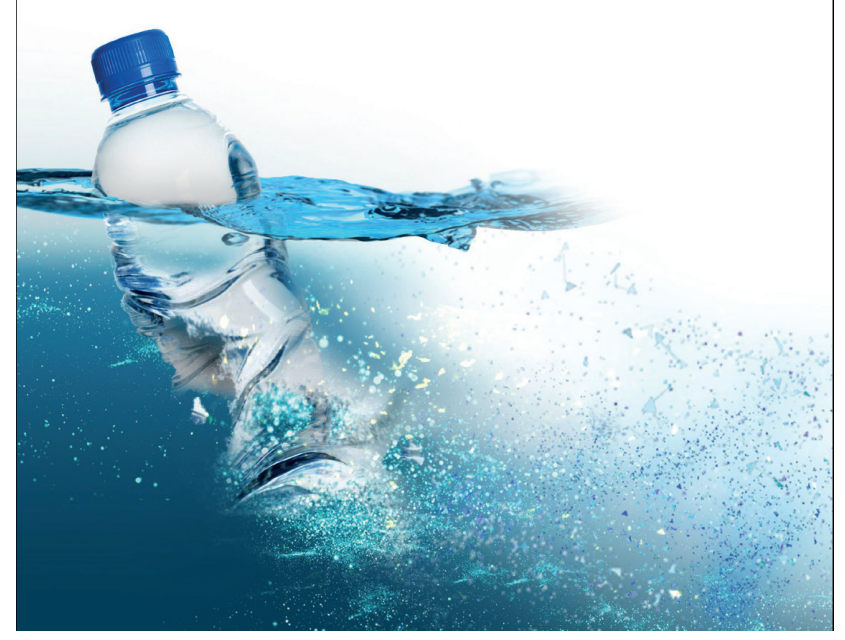

Figure 2. Plastic pollution is produced and generated by many sources of chemical compounds, affecting not only the health of marine organisms but potentially interacting at all molecular levels in the trophic chain (Photo art by Allan Jeffs).

The complexity involved with plastic pollution, as it impacts the entire marine ecosystem, results in a partial and sometimes defective picture of the real nature of the contamination. This main issue is magnified by the lack of standardized procedures for plastic quantification on all size ranges and its detection as chemical components associated to plastic itself.

As young scientists based in a developing country, but sharing nevertheless a common interest in environmental microplastic pollution in the oceans, we found it extremely difficult to resolve and establish a general approach to address this global issue especially as research funding is limited. Obviously this may be a common and global dilemma for most scientific staff, not only in developing countries but also worldwide. We are restricted not only in resources to properly evaluate and characterize the real impact of plastic pollution in our oceans research, but this limitation is intensified by the lack, once again, of an internationally established procedure.

It seems that science and global concern around plastic pollution are more influenced by social networks. As a result, researchers are rushing to publish faster without focussing on setting the basic guidelines to follow. In other words, they are trying to get a "piece of the pie" and get something published in today's hot topic.

We urge local and international, private and governmental institutions to develop standard protocols that can help to compare results in order to understand the effect of plastic pollution in the marine environment.

\section{Bibliographic references}

1. Cressey, D. Bottles, bags, ropes and toothbrushes: the struggle to track ocean plastics. Nature 536, 263-265 (2016).

2. Lebreton, L. et al. Evidence that the Great Pacific Garbage Patch is rapidly accumulating plastic. Sci. Rep. 8, 4666 (2018).

3. Eriksen, M. et al. Plastic Pollution in the World's Oceans: More than 5 Trillion Plastic Pieces Weighing over 250,000 Tons Afloat at Sea. PLoS ONE 9, el11913 (2014).

4. Tanaka, K. \& Takada, H. Microplastic fragments and microbeads in digestive tracts of planktivorous fish from urban coastal waters. Sci. Rep. 6, 768 (2016).

5. Laist, D. W. in link.springer.com 99-139 (Springer New York, 1997). doi:10.1007/978-1-4613-8486-1_10

6. Vega Thurber, R. L. et al. Chronic nutrient enrichment increases prevalence and severity of coral disease and bleaching. Glob Change Biol 20, 544-554 (2013).

7. Mathieu-Denoncourt, J., Wallace, S. J., de Solla, S. R. \& Langlois, V. S. Plasticizer endocrine disruption: Highlighting developmental and reproductive effects in mammals and non-mammalian aquatic species. Gen. Comp. Endocrinol. 219, 74-88 (2015).

8. Christensen, J. H., Glasius, M., Pécseli, M., Platz, J. \& Pritzl, G. Polybrominated diphenyl ethers (PBDEs) in marine fish and blue mussels from southern Greenland. Chemosphere 47, 631-638 (2002).

9. Rohwer, F. \& Thurber, R. V. Viruses manipulate the marine environment. Nature 459, 207-212 (2009).

10. Murphy, S. et al. Reproductive Failure in UK Harbour Porpoises Phocoena phocoena : Legacy of Pollutant Exposure? PLoS ONE 10, e0131085 (2015).

11. Oehlmann, J., Oetken, M. \& Schulte-Oehlmann, U. A critical evaluation of the environmental risk assessment for plasticizers in the freshwater environment in Europe, with special emphasis on bisphenol $A$ and endocrine disruption. Environmental Research 108, 140-149 (2008). 